Buletin Ilmiah Math. Stat. dan Terapannya (Bimaster)

Volume 08, No. 4 (2019), hal 745 - 750.

\title{
BEBERAPA SIFAT KRONECKER PRODUCT
}

\author{
Flasida Masita, Shantika Martha, Fransiskus Fran
}

INTISARI

\begin{abstract}
Operasi pada matriks yang mensyaratkan ukuran diantaranya adalah operasi penjumlahan, operasi pengurangan, dan operasi perkalian. Selain itu terdapat operasi lain yang tidak mensyaratkan ukuran yaitu Kronecker product, yang dilambangkan dengan notasi $\otimes$. Penelitian ini bertujuan untuk mengkaji sifat-sifat Kronecker product pada matriks, diantaranya sifat operasi terkait penjumlahan, perkalian skalar matriks, perkalian matriks, transpos matriks, invers matriks, trace matriks, dan determinan matriks. Pada penelitian ini juga dibahas bahwa matriks hasil Kronecker product dari dua matriks normal, matriks ortogonal, atau matriks simetri berturutturut adalah matriks normal, matriks ortogonal, dan matriks simetri.
\end{abstract}

Kata Kunci : Matriks, Determinan, Invers, Kronecker Product.

\section{PENDAHULUAN}

Matriks adalah susunan persegi panjang dari bilangan-bilangan yang dibatasi dengan tanda kurung. Bilangan-bilangan dalam susunan tersebut dinamakan entri atau elemen dalam matriks. Pada matriks terdapat beberapa operasi yang mensyaratkan ukuran, diantaranya adalah operasi penjumlahan, operasi pengurangan, dan operasi perkalian pada matriks. Selain itu terdapat operasi lain yang tidak mensyaratkan ukuran yaitu Kronecker product. Kronecker product dapat diaplikasikan salah satunya untuk kasus persaman Sylvester dan persamaan Lyapunov yang menyatakan persamaan matriks linear. Pada persamaan Sylvester diperoleh bentuk umumnya adalah $\sum_{i=1}^{k} A_{i} X B_{i}=C$. Persamaan Lyapunov diperoleh bentuk umumnya $A X+X A^{T}=C$, dimana $C$ adalah simetri. Kronecker product dapat diaplikasikan juga untuk model dan algoritma multi arah, varians dalam statistik dan ekonomi, pemrosesan sinyal, serta turunan pada matriks [1].

Pada tahun 1880-an, operasi Kronecker dua buah matriks diperkenalkan oleh matematikawan dari Jerman yang bernama Leopold Kronecker. Salah seorang mahasiswanya, yang bernama Hensel, mempopulerkan notasinya dengan nama Kronecker product [2]. Kronecker product merupakan operasi pada dua matriks yang tidak mensyaratkan ukuran. Kronecker product dilambangkan dengan notasi $\otimes$. Kronecker product dari dua matriks $A$ dan $B$ dimana $A=\left[a_{i j}\right]$ yang berukuran $m \times n$, dengan $i=1,2,3, \ldots, m$ dan $j=1,2,3, \ldots, n$. Untuk $B=\left[b_{s t}\right]$ berukuran $p \times q$, dengan $s=1,2,3, \ldots, p$ dan $t=1,2,3, \ldots, q$. Sehingga dapat dibentuk ke dalam Kronecker Product adalah $A \otimes B=\left[\begin{array}{ccc}a_{11} B & \cdots & a_{1 n} B \\ \vdots & \ddots & \vdots \\ a_{m 1} B & \cdots & a_{m n} B\end{array}\right]$. Matriks dari hasil Kronecker Product $A$ dan $B$ membentuk suatu matriks baru yang berukuran $m p \times n q$ [2].

Pada penelitian ini akan dibahas sifat-sifat Kronecker product pada matriks, langkahlangkah yang dilakukan pada penelitian ini adalah diberikan matriks $A$ dan $B$ kemudian dilakukan operasi-operasi matriks dengan mengkaji sifat-sifat Kronecker product pada matriks, diantaranya sifat operasi terkait penjumlahan, perkalian skalar matriks, perkalian matriks, transpos matriks, invers matriks, trace matriks, dan determinan matriks. Pada penelitian ini juga dibahas bahwa matriks hasil Kronecker product dari dua matriks normal, matriks ortogonal, atau matriks simetri berturut-turut adalah matriks normal, matriks ortogonal, dan matriks simetri. 


\section{MATRIKS}

Matriks adalah susunan segiempat siku-siku dari bilangan-bilangan yang dibatasi dengan tanda kurung. Bilangan-bilangan dalam susunan tersebut dinamakan entri atau elemen dalam matriks[3]. Bentuk umum dari matriks $A_{m \times n}$ adalah:

$$
A_{m \times n}=\left[\begin{array}{cccc}
a_{11} & a_{12} & \cdots & a_{1 n} \\
a_{21} & a_{22} & \cdots & a_{2 n} \\
\vdots & \vdots & \ddots & \vdots \\
a_{m 1} & a_{m 2} & \cdots & a_{m n}
\end{array}\right]
$$

Baris-baris dari matrks $A$ seperti di atas adalah $m$ deret horizontal yang terdiri dari skalar-skalar:

$$
\left[\begin{array}{llll}
a_{11} & a_{12} & \cdots & a_{1 n}
\end{array}\right],\left[\begin{array}{llll}
a_{21} & a_{22} & \cdots & a_{2 n}
\end{array}\right], \ldots,\left[\begin{array}{llll}
a_{m 1} & a_{m 2} & \cdots & a_{m n}
\end{array}\right],
$$

dan kolom-kolom dari matriks $A$ adalah $n$ deretan vertikal yang terdiri dari skalar-skalar:

$$
\left[\begin{array}{c}
a_{11} \\
a_{21} \\
\vdots \\
a_{m 1}
\end{array}\right],\left[\begin{array}{c}
a_{12} \\
a_{22} \\
\vdots \\
a_{m 2}
\end{array}\right], \ldots,\left[\begin{array}{c}
a_{1 n} \\
a_{2 n} \\
\vdots \\
a_{m n}
\end{array}\right]
$$

Perhatikan bahwa elemen $a_{i j}$ disebut entri $i j$ dari matriks $A$ yang terletak pada baris $i$ dan kolom $j$ atau seringkali matriks tersebut ditulis sebagai $A=\left[a_{i j}\right]$. Pada matriks terdapat jenis-jenis matriks berdasarkan ukuran dan elemen yaitu matriks kuadrat, matriks nol, matriks satuan, matrks diagonal, matriks skalar, matriks segitiga bawah, matriks segitiga bawah, matriks simetris, matriks singular, matriks nonsingular, matriks ortogonal, matriks normal, matriks blok.

Jika diketahui suatu matriks $A=\left[a_{i j}\right]$ berukuran $m \times n$ maka transpos dari $A$ adalah matriks $A^{T}$ berukuran $n \times m$ yang didapat dari $A$ dengan menuliskan baris ke- $i$ dari $A$ sebagai kolom ke $-i$ dari $A^{T}$. Sebagai contoh, jika

$A=\left[\begin{array}{lll}a & b & c \\ d & e & f \\ g & h & i\end{array}\right]$ maka $A^{T}=\left[\begin{array}{lll}a & d & g \\ b & e & h \\ c & f & i\end{array}\right]$.

Jumlah unsur-unsur diagonal (diagonal utama) dari suatu matriks persegi $A$ disebut $\operatorname{tr}(A)$. Dari matriks persegi $A_{n \times n}$, diperoleh $\operatorname{tr}(A)$, yaitu:

\section{OPERASI MATRIKS}

$$
\operatorname{tr}(A)=a_{11}+a_{22}+a_{33}+\cdots+a_{n n}
$$

Operasi matriks adalah operasi aljabar terhadap dua atau lebih matriks yang meliputi:

(i). Penjumlahan Matriks

Syarat untuk menjumlahkan dua matriks atau lebih harus mempunyai ordo yang sama. Misalkan matriks $A$ dan $B$ mempunyai ordo $m \times n$ atau dapat ditulis $=\left[a_{i j}\right]_{m \times n}, B=\left[b_{i j}\right]_{m \times n}$ dengan $i=1,2, \ldots, m$ dan $j=1,2,3, \ldots, n$. Jika kedua matriks tersebut dijumlahkan, maka akan terdapat matriks $C=\left[c_{i j}\right]_{m \times n}$, dimana $C=A+B$ dengan $c_{i j}=a_{i j}+b_{i j}$.

Beberapa sifat operasi penjumlahan pada matriks, yaitu sebagai berikut:

i. $A+B=B+A$

ii. $(A+B)+C=A+(B+C)=A+B+C$

iii. $A+0=0+A=A$

iv. $A+(-A)=(-A)+A=0$

(ii). Perkalian matriks dengan skalar

Operasi skalar dengan matriks adalah dengan mengalikan semua elemen-elemen matriks dengan suatu skalar. Jika $k$ adalah suatu bilangan skalar dan $A=\left[a_{i j}\right]$ maka matriks $k A=\left[k a_{i j}\right]$ yaitu suatu matriks $k A$ yang diperoleh dengan mengalikan semua elemen matriks $A$ dengan $k$. 
(iii). Perkalian matriks

Dua buah matriks dapat dikalikan jika memiliki jumlah kolom matriks pertama yang sama dengan jumlah baris matriks ke dua. Ordo matriks hasil perkalian dua matriks adalah jumlah baris pertama dikali jumlah kolom kedua. Matriks $A$ memiliki jumlah kolom sebanyak $m$ dan jumlah baris $r$, matriks $B$ memiliki jumlah kolom sebanyak $r$ dan jumlah baris $m$, hasil perkalian matriks $A$ dan $B$ adalah matriks $C$ dengan jumlah kolom $m$ dan jumlah baris $n$.

$$
A_{m \times r} B_{r \times n}=C_{m \times n}
$$

\section{KRONECKER PRODUCT}

Kronecker product merupakan operasi pada dua matriks yang tidak mensyaratkan ukuran. Kronecker product dilambangkan dengan notasi $\otimes$. Diberikan $\in \mathbb{R}^{m \times n}, B \in \mathbb{R}^{p \times q}$, maka kronecker product dari $A$ dan $B$ didefinisikan sebagai

$A \otimes B=\left[\begin{array}{ccc}a_{11} B & \cdots & a_{1 n} B \\ \vdots & \ddots & \vdots \\ a_{m 1} B & \cdots & a_{m n} B\end{array}\right] \in \mathbb{R}^{m p \times n q}$. Definisi yang sama berlaku jika $A$ dan $B$ adalah matriks dengan elemen bilangan kompleks [5].

Sebagai contohnya, misalkan $A=\left[\begin{array}{lll}3 & 1 & 2 \\ 2 & 4 & 5\end{array}\right]$ dan $B=\left[\begin{array}{ll}1 & 2 \\ 3 & 3\end{array}\right]$, maka

$A \otimes B=\left[\begin{array}{ccc}3 B & B & 2 B \\ 2 B & 4 B & 5 B\end{array}\right]$

$$
=\left[\begin{array}{cccccc}
3 & 6 & 1 & 2 & 2 & 4 \\
9 & 9 & 3 & 3 & 6 & 6 \\
2 & 4 & 4 & 8 & 5 & 10 \\
6 & 6 & 12 & 12 & 15 & 15
\end{array}\right]
$$

dan,

$$
\begin{aligned}
B \otimes A=\left[\begin{array}{cc}
A & 2 A \\
3 A & 3 A
\end{array}\right] & \\
= & {\left[\begin{array}{cccccc}
3 & 1 & 2 & 6 & 2 & 4 \\
2 & 4 & 5 & 4 & 8 & 10 \\
9 & 3 & 6 & 9 & 3 & 6 \\
6 & 12 & 15 & 6 & 12 & 13
\end{array}\right] }
\end{aligned}
$$

Oleh karena itu diperoleh bahwa $B \otimes A \neq A \otimes B$.

\section{SIFAT-SIFAT KRONECKER PRODUCT}

Diberikan beberapa sifat dari Kronecker product adalah sebagai berikut:

Teorema 1 Misalkan $A \in \mathbb{R}^{m \times n}$ dan $B \in \mathbb{R}^{p \times q}, C \in \mathbb{R}^{r \times s}$ maka,

i. $A \otimes(B+C)=(A \otimes B)+(A \otimes C)$.

ii. (kA) $\otimes B=A \otimes k B=k(A \otimes B)$.

iii. $(A \otimes B) \otimes C=A \otimes(B \otimes C)$

\section{Bukti:}

Akan dibuktikan untuk (i) dan (ii). Untuk (iii) dapat dibuktikan dengan cara yang serupa.

$$
\text { (i) } A \otimes(B+C)=\left[\begin{array}{ccc}
a_{11}(B+C) & \cdots & a_{1 n}(B+C) \\
\vdots & \ddots & \vdots \\
a_{n 1}(B+C) & \cdots & a_{m n}(B+C)
\end{array}\right]
$$




$$
\begin{aligned}
=\left[\begin{array}{ccc}
a_{11} B+a_{11} C & \cdots & a_{1 n} B+a_{1 n} C \\
\vdots & \ddots & \vdots \\
a_{n 1} B+a_{n 1} C & \cdots & a_{n n} B+a_{n n} C
\end{array}\right] & =\left[\begin{array}{ccc}
a_{11} B & \cdots & a_{1 n} B \\
\vdots & \ddots & \vdots \\
a_{m 1} & \cdots & a_{m n} B
\end{array}\right]+\left[\begin{array}{ccc}
a_{11} C & \cdots & a_{1 n} C \\
\vdots & \ddots & \vdots \\
a_{m 1} C & \cdots & a_{m n} C
\end{array}\right] \\
& =(A \otimes B)+(A \otimes C) .
\end{aligned}
$$

(ii) $(k A) \otimes B=A \otimes k B=k(A \otimes B)$.

$(\mathrm{kA}) \otimes B=\left[\begin{array}{ccc}k a_{11} B & \cdots & k a_{1 n} B \\ \vdots & \ddots & \vdots \\ k a_{m 1} B & \cdots & k a_{m n} B\end{array}\right]=\left[\begin{array}{ccc}a_{11} k B & \cdots & a_{1 n} k B \\ \vdots & \ddots & \vdots \\ a_{m 1} k B & \cdots & a_{m n} k B\end{array}\right]=A \otimes(k B)$

$A \otimes(k B)=\left[\begin{array}{ccc}a_{11} k B & \cdots & a_{1 n} k B \\ \vdots & \ddots & \vdots \\ a_{m 1} k B & \cdots & a_{m n} k B\end{array}\right]=\left[\begin{array}{ccc}k a_{11} B & \cdots & k a_{1 n} B \\ \vdots & \ddots & \vdots \\ k a_{m 1} B & \cdots & k a_{m n} B\end{array}\right]=k\left[\begin{array}{ccc}a_{11} B & \cdots & a_{1 n} B \\ \vdots & \ddots & \vdots \\ a_{m 1} B & \cdots & a_{m n} B\end{array}\right] k(A \otimes B)$

Teorema 2 Diberikan $A \in \mathbb{R}^{m \times n}, B \in \mathbb{R}^{r \times s}, C \in \mathbb{R}^{n \times p}$, dan $D \in \mathbb{R}^{s \times t}$.

Maka $(A \otimes B)(C \otimes D)=A C \otimes B D \in \mathbb{R}^{m r \times p t}$.

Bukti:

$$
\begin{aligned}
(A \otimes B)(C \otimes D) & =\left[\begin{array}{ccc}
a_{11} B & \cdots & a_{1 n} B \\
\vdots & \ddots & \vdots \\
a_{m 1} B & \cdots & a_{m n} B
\end{array}\right]\left[\begin{array}{ccc}
c_{11} D & \cdots & c_{1 p} D \\
\vdots & \ddots & \vdots \\
c_{n 1} D & \cdots & c_{n p} D
\end{array}\right] \\
& =\left[\begin{array}{cccc}
\sum_{k=1}^{n} a_{1 k} c_{k 1} B D & \cdots & \sum_{k=1}^{n} a_{1 k} c_{k p} B D \\
\vdots & \ddots & \vdots \\
\sum_{k=1}^{n} a_{m k} c_{k 1} B D & \cdots & \sum_{k=1}^{n} a_{m k} c_{k p} B D
\end{array}\right] \\
& =A C \otimes B D .
\end{aligned}
$$

Akibat 3 Jika $A \in \mathbb{R}^{n \times n}$ dan $B \in \mathbb{R}^{m \times m}$ maka $A \otimes B=\left(A \otimes I_{m}\right)\left(I_{n} \otimes B\right)$.

Bukti : Berdasarkan Teorema 2 maka $\left(A \otimes I_{m}\right)\left(I_{n} \otimes B\right)=\left(A I_{n}\right) \otimes\left(I_{m} B\right)=A \otimes B$.

Teorema 4 Jika $A \in \mathbb{R}^{m \times n}$ dan $B \in \mathbb{R}^{p \times q}$ maka $(A \otimes B)^{T}=A^{T} \otimes B^{T}$.

Bukti:

$$
\begin{aligned}
& (A \otimes B)=\left[\begin{array}{ccc}
a_{11} B & \cdots & a_{1 n} B \\
\vdots & \ddots & \vdots \\
a_{m 1} B & \cdots & a_{m n} B
\end{array}\right] \\
& =\left[\begin{array}{cccccc}
a_{11} b_{11} & \cdots & a_{11} b_{1 q} & a_{1 n} b_{11} & \cdots & a_{1 n} b_{1 q} \\
\vdots & \ddots & \vdots & \vdots & \ddots & \vdots \\
a_{11} b_{p 1} & \cdots & a_{11} b_{p q} & a_{1 n} b_{p 1} & \cdots & a_{1 n} b_{p q} \\
a_{m 1} b_{11} & \cdots & a_{m 1} b_{1 q} & a_{m n} b_{11} & \cdots & a_{m n} b_{1 q} \\
\vdots & \ddots & \vdots & \vdots & \ddots & \vdots \\
a_{m 1} b_{p 1} & \cdots & a_{m 1} b_{p q} & a_{m n} b_{p 1} & \cdots & a_{m n} b_{p q}
\end{array}\right] \\
& (A \otimes B)^{T}=\left[\begin{array}{cccccc}
a_{11} b_{11} & \cdots & a_{11} b_{p 1} & a_{m 1} b_{11} & \cdots & a_{m 1} b_{p 1} \\
\vdots & \ddots & \vdots & \vdots & \ddots & \vdots \\
a_{11} b_{1 q} & \cdots & a_{11} b_{p q} & a_{m 1} b_{1 q} & \cdots & a_{m 1} b_{p q} \\
a_{1 n} b_{11} & \cdots & a_{1 n} b_{p 1} & a_{m n} b_{11} & \cdots & a_{m n} b_{p 1} \\
\vdots & \ddots & \vdots & \vdots & \ddots & \vdots \\
a_{1 n} b_{1 q} & \cdots & a_{1 n} b_{p q} & a_{m n} b_{1 q} & \cdots & a_{m n} b_{p q}
\end{array}\right] \\
& =\left[\begin{array}{ccc}
a_{11} B^{T} & \cdots & a_{m 1} B^{T} \\
\vdots & \ddots & \vdots \\
a_{1 n} B^{T} & \cdots & a_{m n} B^{T}
\end{array}\right]=A^{T} \otimes B^{T}
\end{aligned}
$$


Akibat 5 Jika $A \in \mathbb{R}^{n \times n}$ dan $B \in \mathbb{R}^{m \times m}$ matriks simetris, maka $A \otimes B$ juga matriks simetris.

Bukti : Misalkan $A$ dan $B$ matriks simetris, maka $A=A^{T}$ dan $B=B^{T}$. Akibatnya berdasarkan Teorema $4,(A \otimes B)^{T}=A^{T} \otimes B^{T}=A \otimes B$.

Teorema 6 Jika $A$ dan $B$ adalah matriks non singular, maka $(A \otimes B)^{-1}=A^{-1} \otimes B^{-1}$.

Bukti: Menggunakan Teorema $1,(A \otimes B)\left(A^{-1} \otimes B^{-1}\right)=A A^{-1} \otimes B B^{-1}=I \otimes I=I$.

Teorema 7 Jika $A \in \mathbb{R}^{n \times n}$ dan $B \in \mathbb{R}^{m \times m}$ matriks normal, maka $A \otimes B$ juga matriks normal.

Bukti: Misalkan $A$ dan $B$ matriks normal, maka $A^{T} A=A A^{T}$ dan $B^{T} B=B B^{T}$. Akibatnya,

$(A \otimes B)^{T}(A \otimes B)=\left(A^{T} \otimes B^{T}\right)(A \otimes B)=A^{T} A \otimes B^{T} B=A A^{T} \otimes B B^{T}=(A \otimes B)(A \otimes B)^{T}$.

Akibat 8 Jika $A \in \mathbb{R}^{n \times n}$ adalah ortogonal dan $B \in \mathbb{R}^{m \times m}$ adalah ortogonal, maka $A \otimes B$ adalah ortogonal.

Bukti : Diketahui $A$ dan $B$ ortogonal, maka $A^{-1}=A^{T}$ dan $B^{-1}=B^{T}$. maka,

$(A \otimes B)^{T}=A^{T} \otimes B^{T}=A^{-1} \otimes B^{-1}=(A \otimes B)^{-1}$.

Akibat 9 Jika $A \in \mathbb{R}^{n \times n}$ dan $B \in \mathbb{R}^{m \times m}$, maka

1. $\operatorname{tr}(A \otimes B)=(\operatorname{tr} A)(\operatorname{tr} B)=\operatorname{tr}(B \otimes A)$

2. $\operatorname{det}(A \otimes B)=(\operatorname{det} A)^{m}(\operatorname{det} B)^{n}=\operatorname{det}(B \otimes A)$

Bukti :

(i) $(A \otimes B)=\left[\begin{array}{ccc}a_{11} & \cdots & a_{1 n} \\ \vdots & \ddots & \vdots \\ a_{n 1} & \cdots & a_{n n}\end{array}\right] \otimes\left[\begin{array}{ccc}b_{11} & \cdots & b_{1 m} \\ \vdots & \ddots & \vdots \\ b_{m 1} & \cdots & b_{m m}\end{array}\right]$

$$
=\left[\begin{array}{ccccccc}
a_{11} b_{11} & \ldots & a_{11} b_{1 m} & & a_{1 n} b_{11} & \ldots & a_{1 n} b_{1 m} \\
\vdots & \ddots & \vdots & \ldots & \vdots & \ddots & \vdots \\
a_{11} b_{m 1} & \ldots & a_{11} b_{m m} & & a_{1 n} b_{m 1} & \ldots & a_{1 n} b_{m m} \\
& \vdots & & \ddots & & \vdots & \\
a_{n 1} b_{11} & \cdots & a_{n 1} b_{1 m} & & a_{n n} b_{11} & \ldots & a_{n n} b_{1 m} \\
\vdots & \ddots & \vdots & \cdots & \vdots & \ddots & \vdots \\
a_{n 1} b_{m 1} & \ldots & a_{n 1} b_{m m} & & a_{n 1} b_{m 1} & \cdots & a_{n n} b_{m m}
\end{array}\right]
$$

$\operatorname{tr}(A \otimes B)=a_{11} b_{11}+\cdots+a_{11} b_{m m}+\cdots+a_{n n} b_{11}+\cdots+a_{n n} b_{m m}$.

Disisi lain diketahui bahwa $\operatorname{tr}(A)=a_{11}+\cdots+a_{n n}$ dan $\operatorname{tr}(B)=b_{11}+\cdots+b_{m m}$. Akibatnya, $(\operatorname{tr} A)(\operatorname{tr} B)=a_{11} b_{11}+\cdots+a_{11} b_{m m}+\cdots+a_{n n} b_{11}+\cdots+a_{n n} b_{m m}$. Selanjutnya

$B \otimes A=\left[\begin{array}{ccccccc}b_{11} a_{11} & \ldots & b_{11} a_{1 n} & & b_{1 m} a_{11} & \ldots & b_{1 m} a_{1 n} \\ \vdots & \ddots & \vdots & \ldots & \vdots & \ddots & \vdots \\ b_{11} a_{n 1} & \ldots & b_{11} a_{n n} & & b_{1 m} a_{n 1} & \ldots & b_{1 m} a_{n n} \\ & \vdots & & \ddots & & \vdots & \\ b_{m 1} a_{11} & \cdots & b_{m 1} a_{1 n} & & b_{m m} a_{11} & \ldots & b_{m m} a_{1 n} \\ \vdots & \ddots & \vdots & \ldots & \vdots & \ddots & \vdots \\ a_{n 1} & \ldots & b_{m 1} a_{n n} & & b_{m m} a_{n 1} & \cdots & b_{m m} a_{n n}\end{array}\right]$

$\operatorname{tr}(B \otimes A)=b_{11} a_{11}+\cdots+b_{11} a_{n n}+\cdots+b_{m m} a_{11}+\cdots+b_{m m} a_{n n}$.

Jadi dapat disimpulkan bahwa $\operatorname{tr}(A \otimes B)=(\operatorname{tr} A)(\operatorname{tr} B)=\operatorname{tr}(B \otimes A)$

(ii) Berdasarkan Akibat 3 maka $A \otimes B=\left(A \otimes I_{m}\right)\left(I_{n} \otimes B\right)$. Sehingga,

$|A \otimes B|=\left|\left(A \otimes I_{m}\right)\left(I_{n} \otimes B\right)\right|=\left|A \otimes I_{m}\right|\left|I_{n} \otimes B\right|$

$$
=\left|\begin{array}{ccc}
a_{11} I_{m} & \cdots & a_{1 n} I_{m} \\
\vdots & \ddots & \vdots \\
a_{n 1} I_{m} & \cdots & a_{m n} I_{m}
\end{array}\right|\left|\begin{array}{ccc}
B & \cdots & 0 \\
\vdots & \ddots & \vdots \\
0 & \cdots & B
\end{array}\right|
$$

Dengan menggunakan operasi baris elementer dan kolom elementer yaitu pertukaran baris dan pertukaran kolom maka diperoleh $\left|\begin{array}{ccc}a_{11} I_{m} & \ldots & a_{1 n} I_{m} \\ \vdots & \ddots & \vdots \\ a_{n 1} I_{m} & \cdots & a_{m n} I_{m}\end{array}\right|=\left|\begin{array}{ccc}A & \ldots & 0 \\ \vdots & \ddots & \vdots \\ 0 & \ldots & A\end{array}\right|$. 
Akibatnya, $|A \otimes B|=\left|\begin{array}{ccc|ccc}A & \ldots & 0 \\ \vdots & \ddots & \vdots \\ 0 & \ldots & A\end{array}\right|\left|\begin{array}{ccc}B & \ldots & 0 \\ \vdots & \ddots & \vdots \\ 0 & \ldots & B\end{array}\right|=(|A| \ldots|A|)(|B| \ldots|B|)=|A|^{m}|B|^{n}$.

\section{KESIMPULAN}

Pada penelitian ini dapat disimpulkan bahwa beberapa sifat Kronecker products pada matriks adalah sebagai berikut:

1. Untuk $A \in \mathbb{R}^{m \times n}$ dan $B \in \mathbb{R}^{p \times q}, C \in \mathbb{R}^{r \times s}$ maka:

i. $A \otimes(B+C)=(A \otimes B)+(A \otimes C)$

ii. $(\mathrm{kA}) \otimes B=A \otimes k B=k(A \otimes B)$

iii. $(A \otimes B) \otimes C=A \otimes(B \otimes C)$

2. Untuk $A \in \mathbb{R}^{m \times n}, B \in \mathbb{R}^{r \times s}, C \in \mathbb{R}^{n \times p}$, dan $D \in \mathbb{R}^{s \times t}$, maka $(A \otimes B)=(C \otimes D)=A C \otimes B D \in$ $\mathbb{R}^{m r \times p t}$.

3. Jika $A \in \mathbb{R}^{n \times n}$ dan $B \in \mathbb{R}^{m \times m}$ maka $A \otimes B=\left(A \otimes I_{m}\right)\left(I_{n} \otimes B\right)$

4. Untuk semua $A$ dan $B$, jika $A \in \mathbb{R}^{m \times n}$ dan $B \in \mathbb{R}^{p \times q}$ maka $(A \otimes B)^{T}=A^{T} \otimes B^{T}$.

5. Jika $A \in \mathbb{R}^{n \times n}$ dan $B \in \mathbb{R}^{m \times m}$ matriks simetris, maka $A \otimes B$ juga matriks simetris.

6. Jika $A$ dan $B$ adalah matriks non singular, maka $(A \otimes B)^{-1}=A^{-1} \otimes B^{-1}$.

7. Jika $A \in \mathbb{R}^{n \times n}$ dan $B \in \mathbb{R}^{m \times m}$ matriks normal, maka $A \otimes B$ juga matriks normal.

8. Jika $A \in \mathbb{R}^{n \times n}$ adalah ortogonal dan $B \in \mathbb{R}^{m \times m}$ adalah ortogonal, maka $A \otimes B$ adalah ortogonal

9. Jika $A \in \mathbb{R}^{n \times n}$ dan $B \in \mathbb{R}^{m \times m}$, maka

i. $\operatorname{tr}(A \otimes B)=(\operatorname{tr} A)(\operatorname{tr} B)=\operatorname{tr}(B \otimes A)$

ii. $\operatorname{det}(A \otimes B)=(\operatorname{det} A)^{m}(\operatorname{det} B)^{n}=\operatorname{det}(B \otimes A)$

\section{DAFTAR PUSTAKA}

[1] Schacke, Kathrin. "On the Kronecker Product".

https://www.math.uwaterloo.ca/ hwolkwi/henry/reports/kronthesissehaec04.pdf, diakses 1 agustus. 2013.

[2] Zhang, Huamin dan Feng Ding. "On the Kronecker Products and Their Applications." Jurnal Matematika Terapan, Article ID 296185, 8 halaman. China: Hindawi Publishing Corporation. 2013; 2 (2).

[3] Kusumawati Ririen. Aljabar Linear dan Matriks. Malang: UIN-Maliki Press. 2014.

[4] Sutojo. T, dkk. Teori dan Aplikasi Aljabar Linear dan Matriks. Yogyakarta: C.V. Andi Offset. 2009.

[5] Laub, Alan J. Matrix Analysis for Scientists and Enginers. Philadelphia: Includes Bibliographical References and Index. 1948.

FLASIDA MASITA

SHANTIKA MARTHA

FRANSISKUS FRAN
: Jurusan Matematika FMIPA UNTAN, Pontianak flasidamasita@gmail.com : Jurusan Matematika FMIPA UNTAN, Pontianak shantika.martha@math.untan.ac.id

: Jurusan Matematika FMIPA UNTAN, Pontianak, fransiskusfran@math.untan.ac.id 